\title{
Phase Behavior of Ternary Blends of Tactic Poly(methyl methacrylate)s and Poly(styrene-co-acrylonitrile)
}

\author{
Wen-Ping $\mathrm{Hsu}^{\dagger}$ and Ching-Fen YeH \\ Department of Applied Chemistry, Chia-Nan College of Pharmacy and Science, \\ \#60 Sec. 1 Erh-Jen Road, Jen-Te Hsian, Tainan, \\ Taiwan 71710, Republic of China
}

(Received October 2, 1998)

\begin{abstract}
Previously, atactic and syndiotactic poly(methyl methacrylate)s (PMMAs) were found to be miscible with poly(styrene-co-acrylonitrile) (PSAN) because all the prepared films were transparent and showed composition dependent glass transition temperatures $\left(T_{\mathrm{g}} \mathrm{s}\right)$. However, the isotactic PMMA/PSAN blend was immiscible because most of the cast films were translucent and had two glass transition temperatures. Isotactic PMMA is known to be miscible with atactic PMMA according to literature. Atactic PMMA is miscible with both PSAN and isotactic PMMA but PSAN and isotactic PMMA are immiscible. It will be interesting to study the miscibility of ternary blends of isotactic and atactic PMMAs and PSAN. An investigation based on this is reported in this article. Calorimetry was used as the principal tool to study miscibility. A blend composed of isotactic and atactic PMMAs was also prepared and confirmed to be miscible. $T_{\mathrm{g}}$ of this blend seemed to vary linearly with composition. An approximate phase diagram of the ternary blends was established based on differential scanning calorimetry data. The results indicated that the ternary blends rich in isotactic PMMA and with a low PSAN concentration were found to be immiscible. One possible cause is attributed to more repulsive MMA-styrene interaction and/or MMA-acrylonitrile (AN) interaction between isotactic PMMA and PSAN.
\end{abstract}

KEY WORDS Tacticity/ Poly(methyl methacrylate)s / Poly(styrene-co-acrylonitrile) / Ternary Blends /

Ternary blends are gaining importance in the field of polymers. The first systematic study on ternary blends was reported by Kwei et al. ${ }^{1}$ in 1977 . In their study, the addition of poly(vinylidene fluoride) (PVDF) to the immiscible pair poly(methyl methacrylate) (PMMA)/ poly(ethyl methacrylate) (PEMA) was studied and found to be miscible. A list of ternaries investigated has been considerably enlarged. ${ }^{2-8}$ In nearly all these blends, a third component either a homopolymer or copolymer is added to homogenize an immiscible pair. Miscibility is often achieved in cases where this third component is miscible with other polymers.

Recently, Bicakci and $\mathrm{Cakmak}^{9}$ investigated the phase behavior of binary and ternary blends of poly(ethylene naphthalate) (PEN), poly(ether imide) (PEI) and poly(ether ether ketone) (PEEK) using differential scanning calorimetry (DSC) and dynamic mechanical analysis (DMTA) techniques. PEN/PEI and PEI/PEEK binary blends exhibit single glass transition temperatures in full composition range and PEN and PEEK were found immiscible particularly at mid-concentration. When PEI is added to the immiscible PEN/PEEK system, first the blends form two separated PEN-rich and PEEK-rich phases below about $40 \%$ PEI concentration, and above this concentration, the three homopolymers form a miscible phase in the amorphous state exhibiting a single $T_{\mathrm{g}}$. An approximate ternary phase diagram was established based on the DSC and DMTA results.

In a previous study, ${ }^{10}$ isotactic, atactic, and syndiotactic PMMAs (designated as $i$, a, and s-PMMAs) with approximately the same molecular weight were blended with poly(styrene- $c o$-acrylonitrile) (PSAN) (containing $25 \mathrm{wt} \%$ of acrylonitrile) in tetrahydrofuran to cast into films. The glass transition temperatures of the polymers were measured. aPMMA and sPMMA were found miscible with PSAN because all the prepared films were transparent and had a single composition dependent glass transition temperature $\left(T_{\mathrm{g}}\right)$. However, iPMMA is not miscible with PSAN because most of the cast films were translucent and had two $T_{\mathrm{g}} \mathrm{s}$. Since iPMMA is miscible with aPMMA according to the literature ${ }^{11}$ but immiscible with PSAN and aPMMA is miscible with PSAN. Therefore an investigation of the miscibility of ternary blends composed of iPMMA, aPMMA, and PSAN is worthwhile and was pursued in this laboratory. aPMMA is acting as a "cosolvent" between PSAN and iPMMA in this study.

In this article, the ternary blends of iPMMA, aPMMA, and PSAN were prepared in several weight ratios. The glass transition temperatures of the ternary mixtures were determined calorimetrically. A blend of iPMMA and aPMMA was made to confirm miscibility. An approximate phase diagram of the ternary blends was established based on calorimetry data and a single $T_{\mathrm{g}}$ was used as the criterion for determining miscibility. The results indicated that the formation of miscible ternary blends is favored when there is a high content of PSAN and/or aPMMA in the blends.

\section{EXPERIMENTAL}

\section{Materials}

Isotactic and atactic PMMAs (designated as $\mathrm{i}$ and a-PMMAs in this study) were purchased from Polysciences, Inc, Warrington, PA. According to supplier information, the molecular weights $\left(M_{w} s\right)$ of iPMMA and aPMMA are the same about 100000 . The PSAN contained $25 \mathrm{wt} \%$ AN units and was purchased from Aldrich Chemical Company Inc., Milwaukee, WI. The $M_{w}$ value for PSAN is 165000 . iPMMA was mixed with

\footnotetext{
† To whom all correspondence should be addressed (Fax: +886-6-2666411).
} 
aPMMA to form a binary blend in the weight ratios of $1 / 3,1 / 1$, and 3/1. A ternary mixture of iPMMA, aPMMA, and PSAN in different weight ratios was also prepared. The actual compositions of the binary and ternary blends are shown in Tables I and II, respectively.

\section{Film Preparation}

Thin films of binary and ternary blends were made by solution casting onto glass plates. Tetrahydrofuran (THF) was used as solvent for most compositions, but for PMMA toluene was used instead. THF and toluene are A.C.S. reagent purchased from Fisher Scientific, Fair Lawn, New Jersey. The final drying step for all the films took place in a vacuum oven for about one day at $92-125^{\circ} \mathrm{C}$, which was above the glass transition temperatures of the individual polymers. The films were cooled to room temperature slowly by air cooling to make as-cast samples. The as-cast samples were used for DSC studies.

\section{Differential Scanning Calorimetry (DSC)}

Glass transition temperatures $\left(T_{g} \mathrm{~s}\right)$ of the polymer blends were determined by a DuPont 2000 thermal analyzer. The experiments were performed in two consecutive scans in the ambient environment of nitrogen gas at a flowing rate of $100-110 \mathrm{ml} \mathrm{min}^{-1}$. At the end of the first thermal scan, the samples stayed at $200^{\circ} \mathrm{C}$ for $1 \mathrm{~min}$. The samples were quenched to $0^{\circ} \mathrm{C}$ immediately using an ice-water bath and were scanned the second time. The scanning temperature from 30 to $200^{\circ} \mathrm{C}$ and heating rate of $20^{\circ} \mathrm{C} \mathrm{min}^{-1}$ were used in each scan. The samples were quenched quickly to prevent crystallization as detected by DSC. There was no trace of solvent in the films detectable by DSC. The inflection point of the specific heat jump of a thermal scan was taken as the glass transition temperature. The glass transition temperatures determined from the first and second thermal scans were designated as $T_{\mathrm{gsc}}$ and $T_{\mathrm{gq}}$, respectively. Therefore, $T_{\mathrm{gsc}}$ is the $T_{\mathrm{g}}$ of the slowly cooled (as-cast) films and $T_{\mathrm{gq}}$ that of the quenched films.

\section{RESULTS AND DISCUSSION}

We did not characterize the tacticity of PMMA by NMR. A simple estimation of the meso and racemic diad fractions was resorted to. The meso and racemic diad fractions of PMMA were estimated previously ${ }^{12}$ according to Fernandez-Martin et al. ${ }^{13}$ or Allen et al. ${ }^{11}$ The average meso fractions from these two methods are $0.687,0.338$, and 0.093 for iPMMA, aPMMA, and sPMMA, respectively.

\section{Fox and Weight Average Equations}

For polymer blends with weak or no interaction, the Fox equation ${ }^{14}$ seems to predict the glass transition temperature quite well. The Fox equation extended for a ternary mixture is shown

$$
1 / T_{\mathrm{g}}=w_{1} / T_{\mathrm{g} 1}+w_{2} / T_{\mathrm{g} 2}+w_{3} / T_{\mathrm{g} 3}
$$

where $T_{\mathrm{g}}$ is the glass transition temperature of a blend, $w_{1}, w_{2}$, and $w_{3}$ are the weight fractions of polymers $\mathbf{1}$, $\mathbf{2}$, and $\mathbf{3}$, respectively. For a binary mixture, $w_{3}=0$.

The weight average equation for a ternary blend is presented below as in eq 2

$$
T_{\mathrm{g}}=w_{1} T_{\mathrm{g} 1}+w_{2} T_{\mathrm{g} 2}+w_{3} T_{\mathrm{g} 3}
$$

where symbols have the same meanings as in eq 1 and again $w_{3}=0$ for a binary one. The Fox and weight average equations were used to estimate $T_{\mathrm{g}}$ of the binary and ternary blends.

\section{Binary Blend of iPMMA and aPMMA}

The iPMMA/aPMMA blend after preparation appeared transparent indicating possible miscibility. The second thermal scans from $30^{\circ} \mathrm{C}$ to $180^{\circ} \mathrm{C}$ of the same blend are presented in Figure 1. Estimated $T_{\mathrm{gsc}}$ and $T_{\mathrm{gq}}$ of the iPMMA/aPMMA blend are listed in Table I. There is not much difference between most $T_{\mathrm{gsc}}$ and $T_{\mathrm{gq}}$ except for one composition. For iPMMA/aPMMA (75.0/25.0),

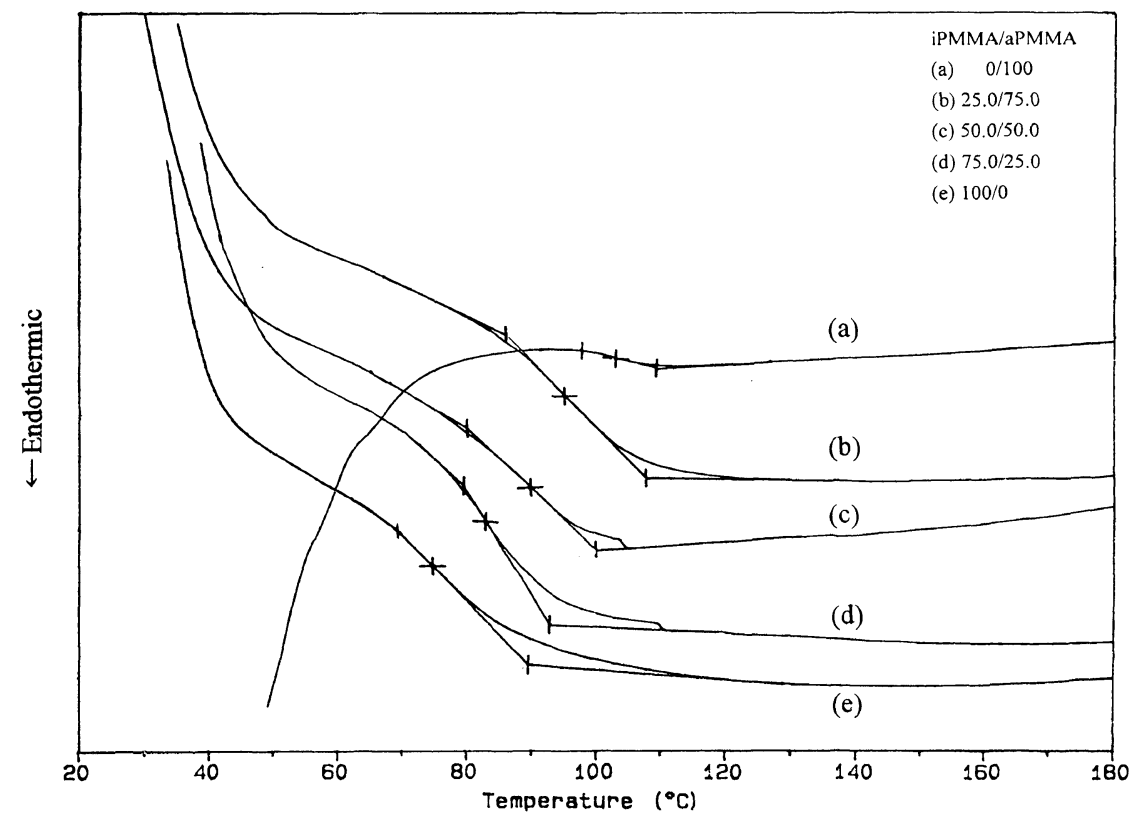

Figure 1. DSC thermograms of the iPMMA/aPMMA blend. 
the as-cast film had two $T_{\mathrm{g}} \mathrm{s}$ but the quenched sample had one $T_{\mathrm{g}}$. Because $T_{\mathrm{gq}}$ are considered to be free of annealing or other thermal/solvent effects, the iPMMA/ aPMMA blend is determined to be miscible in accordance with the literature. ${ }^{11}$ The glass transition temperature regions $\left(\Delta T_{\mathrm{gq}}\right)$ were calculated as differences between the onset and end points of $T_{\mathrm{gq}}$ and are presented in Table I. $\Delta T_{\mathrm{gq}}$ does not show broadening and this can be taken as a sign for good miscibility between iPMMA and aPMMA. Estimated $T_{\mathrm{gq}}$ from the Fox and weight average equations for the iPMMA/aPMMA blend are shown in Table I. It is obvious that the $T_{\mathrm{g}}$ data are fitted well by both equations within experimental error. The results are plotted in Figure 2 and the weight average prediction is shown as the dashed line.

\section{Ternary Blends}

The situation for the ternary blends is certainly not as simple as a binary one. Some compositions of the ternary blends of iPMMA, aPMMA, and PSAN showed some opacity indicating possible immiscibility. The second thermal scans of some compositions of the ternary blends together with iPMMA and PSAN in the same temperature range (as in Figure 1) are shown in Figure 3 for representation. The estimated $T_{\mathrm{gsc}}$ and $T_{\mathrm{gq}}$ for all the studied ternary blends are listed in Table II. The difference between $T_{\mathrm{gsc}}$ and $T_{\mathrm{gq}}$ of most compositions in Table II is small. The only exception is the

Table I. Glass transition temperatures of binary blends

\begin{tabular}{lccccc}
\hline & $T_{\mathbf{g s c}} /{ }^{\circ} \mathrm{C}$ & $T_{\mathbf{g q}} /{ }^{\circ} \mathrm{C}$ & $T_{\mathbf{g}}{ }^{\mathrm{a}}$ & $T_{\mathbf{g}}{ }^{\mathrm{b}}$ & $\Delta T_{\mathbf{g q}} /{ }^{\circ} \mathrm{C}$ \\
\hline iPMMA/aPMMA & & & & \\
100/0 & 71.9 & 74.6 & - & - & 20 \\
$75.0 / 25.0$ & $80.7,98.3$ & 82.4 & 81.2 & 81.6 & 14 \\
$50.0 / 50.0$ & 92.8 & 90.1 & 88.1 & 88.7 & 19 \\
$25.0 / 75.0$ & 94.5 & 95.6 & 95.3 & 95.7 & 21 \\
$0 / 100$ & 103.4 & 102.7 & - & - & 12 \\
\hline
\end{tabular}

${ }^{a}$ Estimated from the Fox equation. ${ }^{b}$ Estimated from weight average.

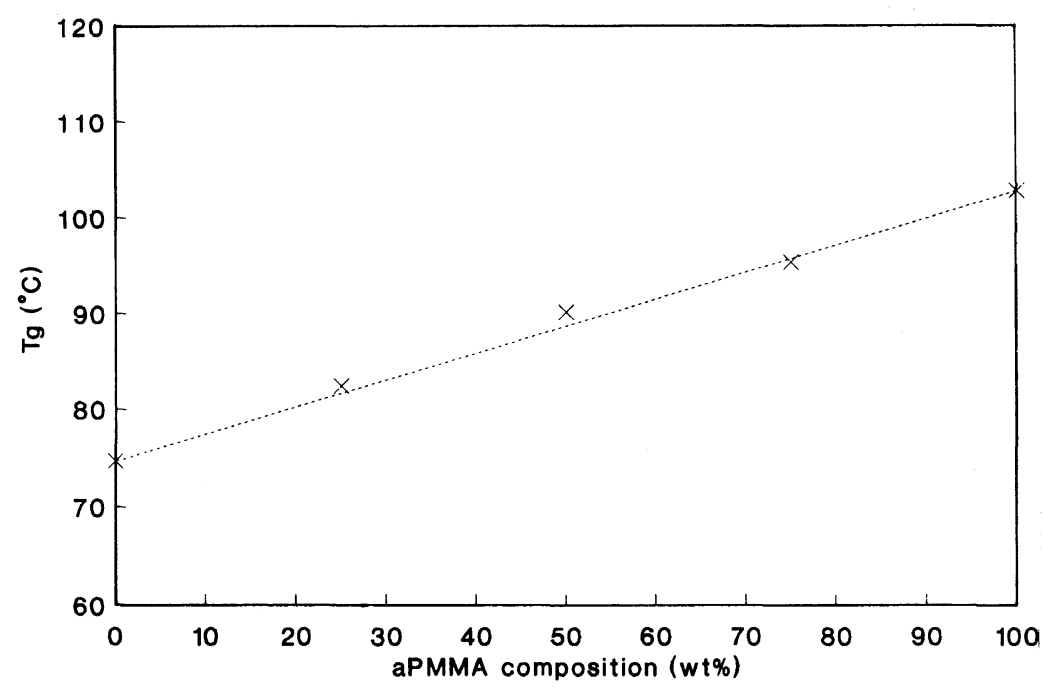

Figure 2. Glass transition temperatures of the iPMMA/aPMMA blend.

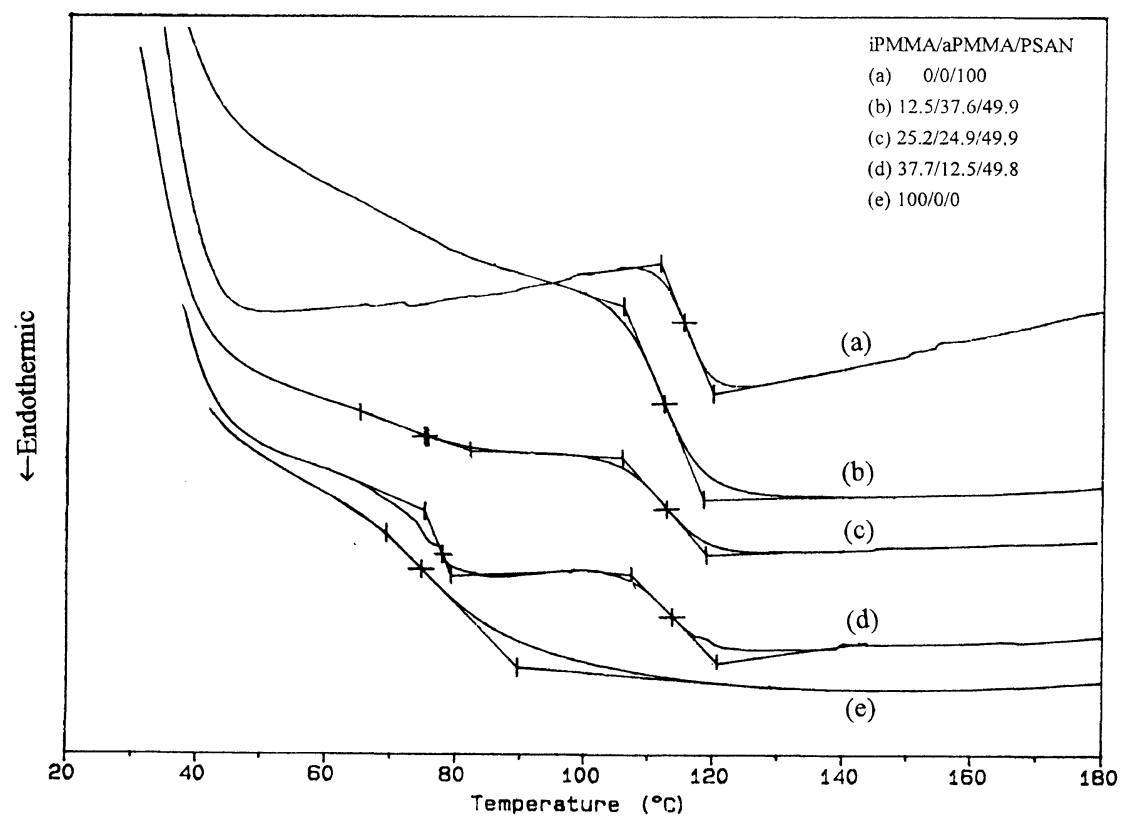

Figure 3. DSC thermograms of the iPMMA/aPMMA/PSAN blend. 
Table II. Glass transition temperatures of ternary blends

\begin{tabular}{lccrcc}
\hline & $T_{\mathrm{gsc}} /{ }^{\circ} \mathrm{C}$ & $T_{\mathrm{gq}} /{ }^{\circ} \mathrm{C}$ & $T_{\mathrm{g}}^{\mathrm{a}}$ & $T_{\mathrm{g}}^{\mathrm{b}}$ & $\Delta T_{\mathrm{gq}} /{ }^{\circ} \mathrm{C}$ \\
\hline iPMMA/aPMMA/PSAN & & & & \\
$100 / 0 / 0$ & 71.9 & 74.6 & - & - & 20 \\
$56.3 / 18.7 / 25.0$ & $72.6,110.8$ & $73.3,108.8$ & 89.1 & 90.0 & 19,11 \\
$37.5 / 37.6 / 24.9$ & $70.9,107.6$ & $78.1,107.7$ & 94.5 & 95.3 & 16,12 \\
$18.7 / 56.3 / 25.0$ & 107.4 & 105.8 & 100.0 & 100.5 & 12 \\
$37.7 / 12.5 / 49.8$ & $72.5,110.0$ & $78.4,113.9$ & 97.3 & 98.3 & 4,13 \\
$25.2 / 24.9 / 49.9$ & $72.1,112.4$ & $76.1,112.9$ & 101.0 & 101.8 & 7,12 \\
$12.5 / 37.6 / 49.9$ & $95.1,112.2$ & 112.7 & 104.9 & 105.4 & 12 \\
$18.8 / 6.3 / 74.9$ & 111.2 & 110.6 & 106.0 & 106.7 & 12 \\
$12.5 / 12.4 / 75.1$ & 109.7 & 110.5 & 108.0 & 108.5 & 11 \\
$6.3 / 19.2 / 74.4$ & 114.6 & 112.9 & 110.2 & 110.1 & 10 \\
$0 / 0 / 100$ & 117.2 & 115.1 & - & - & 9 \\
\hline
\end{tabular}

${ }^{a}$ Estimated from the Fox equation. ${ }^{b}$ Estimated from weight average.

iPMMA/aPMMA/PSAN (12.5/37.6/49.9) blend which showed one $T_{\mathrm{g}}$ after quenching while before having two $T_{\mathrm{g}} \mathrm{s}$. For the same reason as aforementioned, $T_{\mathrm{gq}}$ are used in determining miscibility.

From Table II, the following comments can be made. For the ternary blends with a PSAN composition of $c a$. $25 \%$ or $50 \%$, increasing aPMMA concentration to decrease iPMMA causes the blends to be eventually miscible. The ternary blends with a $c a$. $75 \%$ PSAN are all miscible regardless of the ratio between iPMMA and aPMMA. $T_{\mathrm{g}}$ of the ternaries from either the Fox or weight average equation are tabulated in Table II for reference. For miscible compositions, $T_{\mathrm{g}}$ are always larger than either prediction. For two compositions [iPMMA/aPMMA/PSAN ((12.5/12.4/75.1) and (6.3/ $19.2 / 74.4))]$, weight average predictions are much closer to $T_{\mathrm{g}}$ within $2-3^{\circ} \mathrm{C}$.

For the two compositions [iPMMA/aPMMA/PSAN $((56.3 / 18.7 / 25.0)$ and $(37.5 / 37.6 / 24.9))]$, the situations are quite similar. They both have two $T_{\mathrm{g}} \mathrm{s}$, one $T_{\mathrm{g}}$ close to iPMMA and the other $T_{\mathrm{g}}$ located between aPMMA and PSAN. Therefore for these two compositions, high $T_{\mathrm{g}}$ phase is composed mainly of aPMMA and PSAN and low $T_{\mathrm{g}}$ phase is rich in iPMMA. A subtle difference can be observed from the $T_{\mathrm{g}} \mathrm{s}$ of these two compositions and is stated as follows. Since the PSAN composition is fixed at $c a .25 \%$ for both blends, the addition of aPMMA seems to cause low $T_{\mathrm{g}}$ phase to have a higher $T_{\mathrm{g}}$ and little $T_{\mathrm{g}}$ change of high $T_{\mathrm{g}}$ one. Therefore $T_{\mathrm{g}}$ elevation of the low phase is likely due to the incorporation of aPMMA into this phase. The condition for the other two immiscible compositions (composed about half of PSAN and the ratio between IPMMA and aPMMA being ca. $3 / 1$ and $1 / 1)$ is almost the same. Based on the $T_{\mathrm{g}}$ data, these two ternary blends seem to separate into two phases composed mainly of iPMMA and PSAN, respectively. aPMMA is distributed in these two phases as a minor component causing a lower $T_{\mathrm{g}}$ of the PSAN-rich phase and $T_{\mathrm{g}}$ elevation of the iPMMA-rich phase.

\section{Phase Diagram}

Goh et $a .^{6}$ studied similar but somehow different ternary blends. They investigated the miscibility of PSAN/PMMA/PEMA by DSC. PSAN is known to be both miscible with PMMA and PEMA. However,
Table III. Miscibility of ternary blends

\begin{tabular}{lccc}
\hline PSAN/ca. wt $\%$ & 25 & 50 & 75 \\
\hline aPMMA & + & + & + \\
iPMMA/aPMMA $(c a .1 / 3)$ & + & + & + \\
iPMMA/aPMMA $(c a .1 / 1)$ & - & - & + \\
iPMMA/aPMMA $(c a .3 / 1)$ & - & - & + \\
iPMMA & - & - & + \\
\hline
\end{tabular}

+ , miscible;,- immiscible.

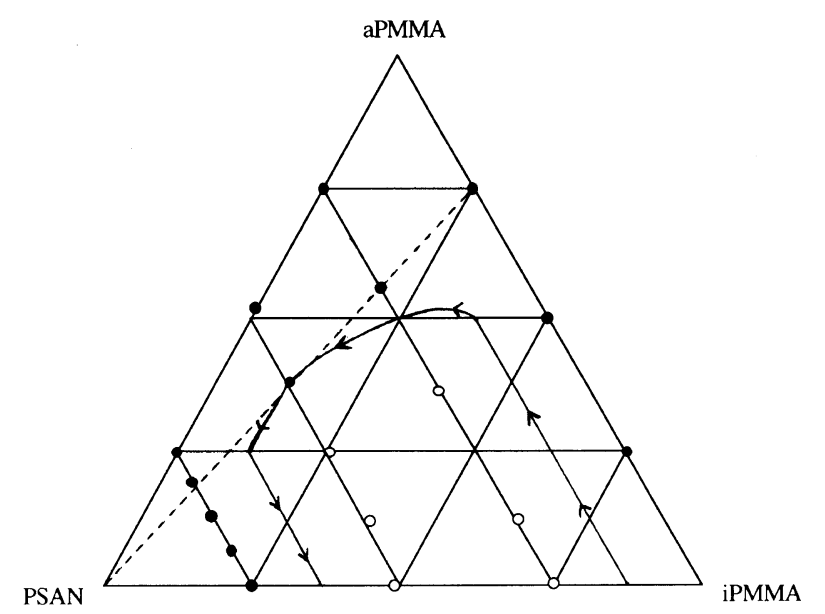

Figure 4. Phase diagram of the iPMMA/aPMMA/PSAN blend. miscible; $\bigcirc$, immiscible.

PMMA and PEMA are not miscible. Therefore PSAN acts as a "cosolvent" between PMMA and PEMA. In their study, they found that the addition of PSAN helps compatibilize the immiscible PMMA/PEMA blends. Our study is different because aPMMA not PSAN is added to homogenize the immiscible pair of PSAN and iPMMA.

The results of Table II and previous data ${ }^{10}$ of iPMMA and aPMMA with PSAN were combined and are listed together in Table III. In this table, PSAN composition and the ratio between $\mathrm{PMMMA} / \mathrm{aPMMA}$ are used as the main indicators. The miscibility is based on the single $T_{\mathrm{g}}$ criterion. The following comments can be drawn from this table. For the ternary blends rich in PSAN compositions $(\mathrm{ca} .75 \%)$, they are all determined to be miscible. The ternary blends with PSAN concentrations at ca. $25 \%$ and $50 \%$ and the ratio between iPMMA and aPMMA $\geq 1$ are immiscible. It is interesting to note that the ternaries with a 1 to 3 ratio between iPMMA and aPMMA are miscible regardless of PSAN composition (shown as the dashed line in Figure 4). The same results can be seen clearly in a phase diagram. A phase diagram of the ternary blends of iPMMA, aPMMA, and PSAN is shown in Figure 4. A postulated phase boundary was established using limited data points. The boundary curve (the curve with arrows in Figure 4) was connected through middle points between miscibility and immiscibility data and one composition [iPMMA/ aPMMA/PSAN (12.5/37.6/49.9)]. This composition can be considered to be borderly miscible because after quenching, two $T_{\mathrm{g}} \mathrm{s}$ become one $T_{\mathrm{g}}$. The boundary curve is rather simplified here but the trend is basically the same even with more data points.

Finally, the reason for ternary blends to be immiscible 
is more complicated than binary blends because of more degrees of freedom and more interactions involved. One possible cause for the ternary blends rich in iPMMA to be immiscible arises from interactions between iPMMA and PSAN. The explanation was given previously ${ }^{10}$ that iPMMA likely forms a more repulsive interaction with styrene or acrylonitrile than aPMMA and sPMMA.

Acknowledgment. The support by the National Science Council of Taiwan, Republic of China Grant NSC-88-2216-E-041-001 is acknowledged and insightful discussion with Prof. T. K. Kwei at Polytechnic University, N.Y. is greatly appreciated.

\section{REFERENCES}

1. T. K. Kwei, H. L. Frisch, W. Radigan, and S. Vogel, Macromolecules, 10, 157 (1977).
2. Y. Y. Wang and S. A. Chen, Polym. Eng. Sci., 21, 47 (1981).

3. D. Rigby, J. L. Lin, and R. J. Roe, Macromolecules, 18, 2269 (1985).

4. V. Shah, J. D. Keitz, D. R. Paul, and J. W. Barlow, J. Appl. Polym. Sci., 32, 3863 (1986).

5. J. I. Equizabal, J. J. Irvin, M. Cotazar, and G. M. Guzman, J. Appl. Polym. Sci., 32, 5945 (1986).

6. S. H. Goh, K. S. Siow, and K. S. Yap, Thermochim. Acta, 105, 191 (1986).

7. S. H. Goh and K. S. Siow, Thermochim. Acta, 102, 281 (1986).

8. W. H. Christiansen, D. R. Paul, and J. W. Barlow, J. Appl. Polym. Sci., 34, 537 (1987).

9. S. Bicakci and M. Cakmak, Polymer, 39, 4001 (1998).

10. W. P. Hsu, J. Appl. Polym. Sci., accepted.

11. P. E. M. Allen, D. M. Host, V. T. Troung, and D. R. G. Williams, Eur. Polym. J., 21, 603 (1985).

12. W. P. Hsu and C. F. Yeh, J. Appl. Polym. Sci., in press.

13. F. Fernandez-Martin, I. Fernandez-Pierola, and A. Horta, J. Polym. Sci. Polym. Phys. Ed., 19, 1353 (1981).

14. T. G. Fox, J. Appl. Bull. Am. Phys. Soc., 1, 123 (1956). 